\title{
An affinity for new proteins
}

Metabolic incorporation of an unnatural amino acid allows researchers to rapidly affinity purify newly synthesized proteins from mammalian cell lysates for direct कo identification by mass spectrometry.

The proteins expressed at any given time in the life cycle of a cell make up its proteomic profile, which is dynamic and ever-changing in response to environmental stimuli. Although mass spectrometry has grown into perhaps the most popular technique for proteomic profiling of different cellular states, it is not always easy to verify which genes are turned on in response to a stimulus because newly synthesized proteins are of course chemically identical to their 'older' siblings.

Hoping to address this problem, Erin Schuman and her colleagues at the California Institute of Technology developed a strategy to directly isolate newly synthesized proteins from cells using the metabolic incorporation of an unnatural amino acid with a bio-orthogonal affinity handle. Using a complementary affinity reagent to isolate the tagged newly synthesized proteins, these 'baby' proteins could be directly identified by mass spectrometry. Improving on the more tradi-
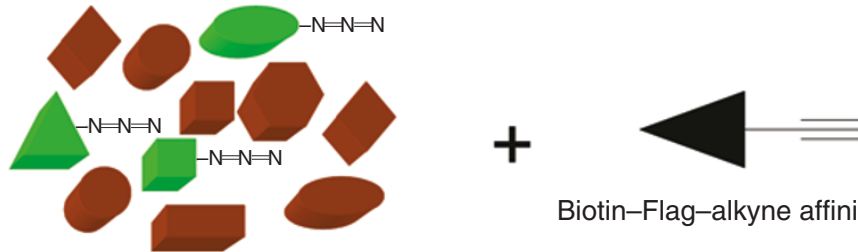

Biotin-Flag-alkyne affinity reagent

Proteomic pool of old and new proteins

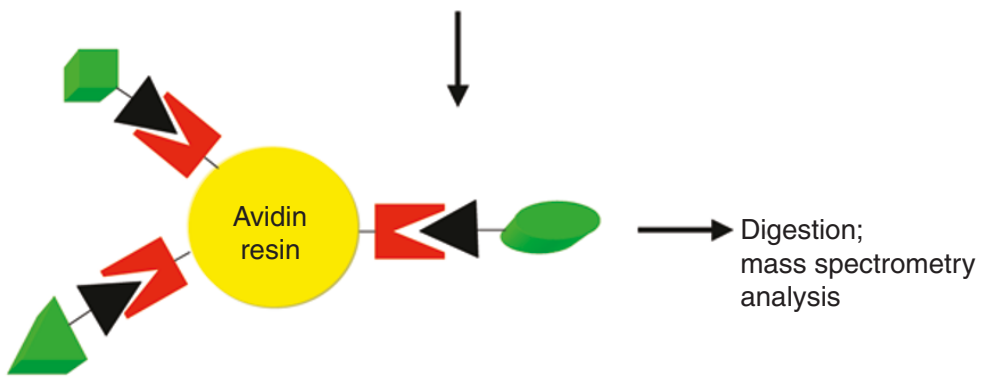

Figure 1 | Overview of the BONCAT strategy. The cell lysate pool containing a 'snapshot' of old and new proteins is incubated with the biotin-Flag-alkyne affinity reagent, which reacts to form a covalent bond to the newly synthesized, azide-labeled proteins. The newly synthesized tagged proteins are pulled out of the lysate with avidin affinity resin and subjected to on-resin digestion and mass spectrometry analysis.

tional method of radioactive tracing with $\left[{ }^{35} \mathrm{~S}\right]$ cysteine or $\left[{ }^{35} \mathrm{~S}\right]$ methionine metabolic incorporation, an affinity handle not only allows the detection of newly synthesized proteins but also their isolation for unambiguous identification.

\section{BIOPHYSICS}

\section{OPTIMIZING YOUR OPTICAL TWEEZERS}

A new measurement theory for dual-trap optical tweezers shows researchers for the first time how to easily optimize their experiments to limit noise from Brownian movement.

The fact that light can be used to physically move objects can be a shocking revelation to those unfamiliar with the concept. In fact, optical tweezers, or analytical optical traps, can be used to measure nanometer scale movements and picoNewton forces. Thus these traps are at the forefront of efforts to understand the innermost workings of single molecules.

To measure forces and movements of molecules using an optical trap, part of a molecule under study is attached to a microsphere that can be held in the trap while another region is attached to a surface or allowed to interact with an immobilized biological molecule. The position and stiffness of the trap can be used to measure movements and forces in the molecular system because the trap acts like a simple spring. Unfortunately, at this scale tiny Brownian movements of the microsphere owing to constant bombardment by the solvent can add considerable noise.

Steve Block previously showed that the physical design of a dual-trap system possessing two independent traps can reduce environmental noise and achieve angstrom-scale resolution, allowing precise measurements of protein movement. Carlos Bustamante and coworkers at the University of California-Berkeley now have developed a theory of measurement that allows them to optimize the performance of this dual-trap design. Graduate student J eff Moffitt says, "The dual-trap design is an excellent way to decouple the instrument from environmental noise." They were concerned, however, that Brownian fluctuations resulting from the solvent would have larger effects on a dual-trap system and wanted to determine how to avoid this.

Bustamante explains the theory that resulted from this inquiry as follows, "When you have two beads you imagine that you are going to have more noise than when you have a single bead because they are uncorrelated and they have their own noise. The subtlety of this analysis is to recognize that the noise between the beads is either in phase or out of phase and only out-of-phase noise can affect your signal. By doing the analysis this way you can basically throw away about $50 \%$ of the noise." Thus, it turns out that the dual-trap instrument is actually less sensitive to Brownian fluctuations than a single trap. 
The unnatural amino acid azidohomoalanine (AHA) is an analog of methionine, which the cell's translational machinery will insert in place of methionine if methionine is left out of the mix. The side chain of AHA contains an azide functional group which is not found in nature, but spontaneously reacts with an alkyne (another functional group not found in nature) to form a covalent bond. To pull out the newly synthesized azide-tagged proteins from the total proteomic pool, Schuman and her colleagues developed an alkyne-based affinity reagent containing a Flag antibody epitope and biotin, which forms an extremely tight noncovalent association with the protein avidin. They entitled their labeling strategy 'BONCAT', or 'bioorthogonal noncanonical amino acid tagging.

As proof of principle, the researchers exposed human embyronic kidney 293 cells to AHA for 2 hours. They used western blotting to verify that AHA was incorporated into a wide range of proteins. They incubated cell lysates with the biotin-Flag-alkyne affinity reagent and used avidin resin to isolate the newly synthesized AHA-tagged proteins. They digested the immobilized proteins on the resin, and subjected the fragments to shotgun mass-spectrometry analysis and database searching (Fig. 1). They successfully identified 1,028 nonredundant peptides, which made up 195 different newly synthesized proteins with a diverse range of functions and biochemistries.

Schuman and her colleagues believe their method provides a unique and simple route to obtaining temporal 'snapshots' of diverse mammalian cellular proteomes. They suggest that BONCAT may be particularly useful for metabolic labeling in post-mitotic cell cultures, in which stable isotope labeling with heavy and light amino acid variants has been challenging.

\section{Allison Doerr}

\section{RESEARCH PAPERS}

Dieterich, D.C. et al. Selective identification of newly synthesized proteins in mammalian cells using bioorthogonal noncanonical amino acid tagging (BONCAT). Proc. Natl. Acad. Sci. USA 103, 9482-9487 (2006).

Their theory allows you to find the experimental signal, defined as a 'generalized coordinate', which will provide the optimal resolution possible within your experimental design parameters such as bead size and trap stiffness that may be constrained for various reasons. This promises to provide researchers with a simple way of getting the best resolution they can at lower spring tensions, which are less likely to negatively affect the system under study.

Coauthor Yann Chemla cautions, "It is important to emphasize that environmental and instrumental sources of noise are still important, and our theory isn't a prescription for getting you high resolution all the time. You really have to build the instrument very carefully and you have to worry about the temperature stability of your room, the mechanical stability of the instrument and even its acoustic isolation."

Now that many labs are considering dual-trap machines for their own experiments, these findings should be a great aid to researchers who want to probe the workings of enzymes and motors at angstrom-level resolution.

\section{Daniel Evanko}

\section{RESEARCH PAPERS}

Moffitt, J R. et al. Differential detection of dual traps improves the spatial resolution of optical tweezers. Proc. Natl. Acad. Sci. USA 103, 9006-9011 (2006).

\section{NEWS IN BRIEF}

\section{CHEMICAL BIOLOGY}

\section{Controlling protein activity with a small molecule- dependent intein}

A primary goal of chemical genetics is to discover a smallmolecule partner for every protein, capable of modulating its activity. As an alternative to extensive small-molecule synthesis and screening, Yuen et al. developed a genetic strategy to control protein function using a 4-hydroxytamoxifendependent, intein-based molecular switch. This molecular switch is particularly attractive for investigating cell-signaling pathways.

Yuen, C.M. et al. J. Am. Chem. Soc. 128, 8939-8946 (2006).

\section{CHROMATIN TECHNIQUES \\ Measuring accessibility of chromosomal DNA on a second time scale}

The mechanism by which proteins find access to DNA packaged in chromatin is a long standing question in the field. Bucceri et al. now have harnessed the light-dependent DNA repair enzyme, photolyase, to monitor the accessibility of specific DNA loci in live cells and at high resolution.

Bucceri, A. et al. EMBO J .; published online 15 J une 2006.

\section{IMAGING AND VISUALIZATION}

\section{A fluorescent nitric-oxide sensor for imaging in live cells} Nitric oxide (NO) is an important cell signaling messenger, but its direct detection in vivo has been extremely difficult. Lim et al. now present a sensor consisting of a copper and fluorescein complex, which upon reaction with NO results in fluorescence, making possible the direct imaging of $\mathrm{NO}$ in live cells. Lim, M.H. et al. Nat. Chem. Biol. 2, 375-380 (2006).

\section{CELL BIOLOGY}

\section{Tumor expression profiling with immuno-LCM}

Buckanovich et al. present a method combining immunohistochemistry and laser-capture microdissection (immuno-LCM) to purify distinct cell populations from complex tissue samples. They used this tool to carefully isolate specific cell populations from tumor microenvironments to facilitate analysis of the underlying molecular events by RNA expression profiling.

Buckanovich, R.J , et al. Cancer Biol. Ther.; published online 9 J une 2006.

\section{MICROSCOPY \\ Probing cell-surface glycans with atomic force microscopy}

There is great general interest from the glycobiology community in new methods for investigating glycan modifications on cell-surface proteins. Using lectins as probes, Lekka et al. used atomic force microscopy to interrogate both the identity of the oligosaccharides as well as their relative densities on the surfaces of living cells.

Lekka, M. et al. Chem. Biol. 13, 505-512 (2006). 